Digestion 1990;46(suppl 2):49

\title{
Synthesis and Degradation of Collagen in Acute
} Experimental Pancreatitis

\begin{tabular}{|l|l|l|}
\hline G. & Adler \\
\hline H.P. & Elsässer \\
\hline H.F. & Kern \\
\hline
\end{tabular}

Departments of Internal Medicine and of Anatomy and Cell Biology, Philipps University of Marburg, FRG 
The mechanisms that lead to pancreatic fibrosis in chronic pancreatitis and pancreatic cancer in man are not known. In particular, there is no information whether fibrosis already starts after a single episode of acute pancreatitis. In the present study the dynamic process of collagen biosynthesis and degradation was analysed in an experimental model of acute interstitial pancreatitis in the rat.

Methods: Pancreatitis was induced in conscious rats by i.v. infusion of the synthetic cholecystokinin analogue caerulein $(10 \mu \mathrm{g} / \mathrm{kg} / \mathrm{h})$ for $12 \mathrm{~h}$. Control animals received saline. Animals of both groups were investigated at the end of infusion and at various time points for up to 10 days. After in vivo labeling with $3 \mathrm{H}$-proline, biosynthesis of collagen was determined $24 \mathrm{~h}$ later by estimation of $3 / 4$-hydroxyproline in pancreatic homogenates. In a second set of experiments $3 / 8$ hydroxyproline was measured 2-7 days after in vivo labeling. For fine structural analysis pancreatic fragments were fixed at different time points.

Results: Two days after induction of pancreatitis an enhanced proliferation of fibroblasts was found. The fibroblasts from controls were spindle-shaped with only few cytoplasmic organelles. After induction of pancreatitis the fibroblasts contained a large Golgi complex and prominent rough endoplasmic reticu-lum. An abundant deposition of collagen fibers oc-cured in areas of destructed pancreatic parenchyma. Ten days after induction of pancreatitis, no increased connective tissue was observed in regenerated pancreas. 24-48 $\mathrm{h}$ after induction of pancreatitis biosynthesis of collagen was increased 15 times when compared to controls. Thereafter, rate of biosynthesis decreased but remained elevated fourfold until day 7. During regeneration the newly snythesized collagen was degraded rapidly.

Conclusion: These data point to an important role of activation of fibroblasts and collagen biosynthesis during regeneration from acute experimental pancreatitis. Collagen deposition was transient and completely degraded after structural reparation of the pancreas. In this experimental model a single episode of pancreatitis did not cause pancreatic fibrosis. 